\title{
Attosecond Control of Orbital Parity Mix Interferences and the Relative Phase of Even and Odd Harmonics in an Attosecond Pulse Train
}

\author{
G. Laurent, ${ }^{*}$ W. Cao, H. Li, Z. Wang, I. Ben-Itzhak, and C. L. Cocke \\ Physics Department, James R. Macdonald Laboratory, Kansas State University, Manhattan, Kansas 66506, USA
}

(Received 19 March 2012; published 20 August 2012)

\begin{abstract}
We experimentally demonstrate that atomic orbital parity mix interferences can be temporally controlled on an attosecond time scale. Electron wave packets are formed by ionizing argon gas with a comb of odd and even high-order harmonics, in the presence of a weak infrared field. Consequently, a mix of energy-degenerate even and odd parity states is fed in the continuum by one- and two-photon transitions. These interfere, leading to an asymmetric electron emission along the polarization vector. The direction of the emission can be controlled by varying the time delay between the comb and infrared field pulses. We show that such asymmetric emission provides information on the relative phase of consecutive odd and even order harmonics in the attosecond pulse train.
\end{abstract}

DOI: 10.1103/PhysRevLett.109.083001

PACS numbers: $32.80 . \mathrm{Rm}, 32.80 . \mathrm{Qk}, 42.65 . \mathrm{Ky}$

Coherent control of electron dynamics in atoms and molecules is a fascinating perspective in laser physics, with promising implications for many different branches of scientific and engineering research. The recent development of $\mathrm{x}$ - or extreme-ultraviolet (XUV) light pulses in the attosecond time scale has opened up new avenues for experimentalists to achieve an effective control over electronic dynamics. Both single attosecond pulses (SAPs) and attosecond pulse trains (APTs) emerge as very promising tools to manipulate the electronic charge distribution in molecules [1-3]. Despite these successful proof-ofprinciple experiments, attosecond control of the electron dynamics is, nevertheless, still in its infancy. This is mainly because the usefulness of such attosecond pulses is limited by the degree to which they can be synthesized and characterized. In particular, a precise measurement of the phases of the frequency components is required as they determine the ultimate length and shape of these pulses. The literature is rich with experimental studies of the characteristics of APTs and SAPs [4-11]. Most of them are based on the conversion of the attosecond pulse into electron wave packets, through photoionization of atoms, in the presence of an IR field to get access to the relative phases of the frequency components making up the pulse.

This scheme, in turn, can be employed to control the electron emission from atoms and molecules. Mauritsson et al. reported an electron scattering imaging technique, based on a sequence of attosecond pulses used to release electrons into a sufficiently strong IR field to guide them back to their parent ions exactly once per laser cycle [12]. A recent theoretical study also suggested combining an APT and a much weaker IR field than in the previous study, as an efficient means for generating strong asymmetric emission of continuum electrons along the direction of the laser polarization [13]. The authors showed that interference between one- and two-photon transitions can produce a large asymmetry in the angular distribution of the photoelectrons though the separate contributions of the two paths have no asymmetries.

In this Letter, we present experimental evidence for such asymmetric emission from an APT formed by odd and even high-order harmonics. We demonstrate that the asymmetric electron emission from atomic targets induced by these APTs in the presence of a weak IR field can be controlled on an attosecond time scale by varying the time delay between both pulses. We show that such asymmetric emission is also related to the relative phases of the harmonics in the comb, allowing, for the first time, the measurement of the phase difference between consecutive odd and even order harmonics. We find the previously unreported result that the relative phase shift between consecutive odd and even harmonics in the plateau region appears to be near

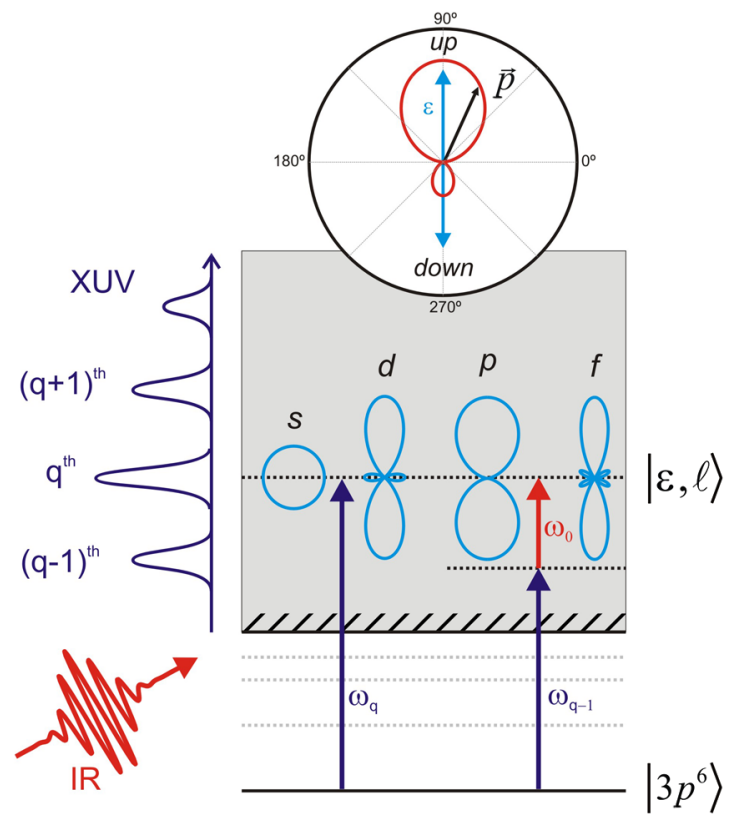

FIG. 1 (color online). Principle of the measurement. 
$\pi / 2$. Our result contradicts the generally accepted physical picture that the combination of even and odd harmonics in the train necessarily creates a series of pulses which occur only once per IR cycle. This picture holds only if there is no phase shift between even and odd harmonics. For a phase shift near $\pi / 2$, the resulting APT has a more complex structure not resembling a single AP once per IR period.

The principle of our experiment is presented in Fig. 1. Argon atoms are ionized using an APT comprised of a comb of odd and even high-order harmonics, in the presence of a weak IR field. Starting from the $3 p^{6}$ ground state, absorption of one XUV photon of frequency $\omega_{q}$ leads to an electron wave packet in $s$ and $d$ orbital states. By absorption of one XUV photon of frequency $\omega_{q-1}\left(\omega_{q+1}\right)$ plus absorption (emission) of one IR photon, $p$ and $f$ states are populated. Consequently, a mix of energy degenerate even $(s, d)$ and odd $(p, f)$ parity states is fed in the continuum. The opposite parity amplitudes interfere. At some appropriate time delay between the comb and IR fields, the even and odd angular continuum wave function resulting from, respectively, the one- and two-photon transitions add constructively on one side (up) of the polarization vector direction and destructively on the other side (down), thus creating a strong up-down asymmetry in the angular emission of the photoelectrons. This asymmetry will then oscillate as the time delay is varied. Hereafter we refer to this process as first-order/second-order interference (FSI).

Our experiment was performed with a 40-fs, $800-\mathrm{nm}$ Ti: sapphire laser. The setup combines an XUV-IR interferometer and a velocity map imaging system [14]. Part of the incoming linearly polarized IR beam was focused and frequency doubled in a $140-\mu \mathrm{m}$-thick $\beta$-barium borate (BBO) crystal. The resulting orthogonally polarized twocolor field was then sent into a $1.5 \mathrm{~mm}$ windowless gas cell filled with 10 Torr of argon. By combining 800 and $400 \mathrm{~nm}$ for the harmonics generation, odd and even harmonic orders were produced [15-17]. The APT was then filtered by using a spatial aperture $(2 \mathrm{~mm}$ diameter at $0.5 \mathrm{~m})$ and a $200 \mathrm{~nm}$ thick Al thin film to remove harmonics below the 11 th order. A replica of the IR (without the $400 \mathrm{~nm}$ ) was sent into the second arm of the interferometer, whose total length could be changed to vary the time delay $\tau$ between the APT and the IR pulse. Both beams were focused, collinearly recombined, and finally sent into a vacuum chamber containing an effusive argon gas jet. At the focal point, the IR intensity is estimated to be below $10^{11} \mathrm{~W} / \mathrm{cm}^{2}$. A velocity map imaging system was used to measure the photoelectrons' momenta. Electrons detected in the upper (lower) part of the detector are associated with the emission in the up (down) direction along the polarization vector.

Our experiment bears a similarity to the reconstruction of attosecond beating by interference of two-photon transitions (RABBITT) technique used to characterize the temporal profile of APTs $[4,6,18-20]$. In that case, a comb of odd-order harmonics only is used to ionize an atomic target in the presence of a weak IR field. Figures 2(a) and 2(c) present our up and down photoelectron energy spectra measured for argon as a function of $\tau$ when only odd harmonics are present in the APT. Electrons are observed at energies corresponding to one-photon absorption of the odd harmonics, and, located in between, sideband peaks due to two-photon transitions (absorption of one XUV photon plus absorption or emission of one IR photon). Two different quantum paths involving two consecutive harmonics contribute to the same sideband quantum state, and thus interfere. As a consequence, the intensity of each sideband peak oscillates with $\tau$ at twice the frequency of the IR field, as predicted by second-order perturbation theory [19]. By comparing Figs. 2(a) and 2(c), we observe that oscillations of the sidebands associated with electrons emitted in up and down directions are in phase, which means that the electron emission is symmetric along the polarization vector direction, at any time delay between the APT and the IR pulse.

The situation changes when the APT is composed of both even and odd harmonics. Now at the photoelectron energy corresponding to each harmonic, three processes contribute: the direct transition from the harmonic, the RABBITT process and the FSI process. Oscillations in

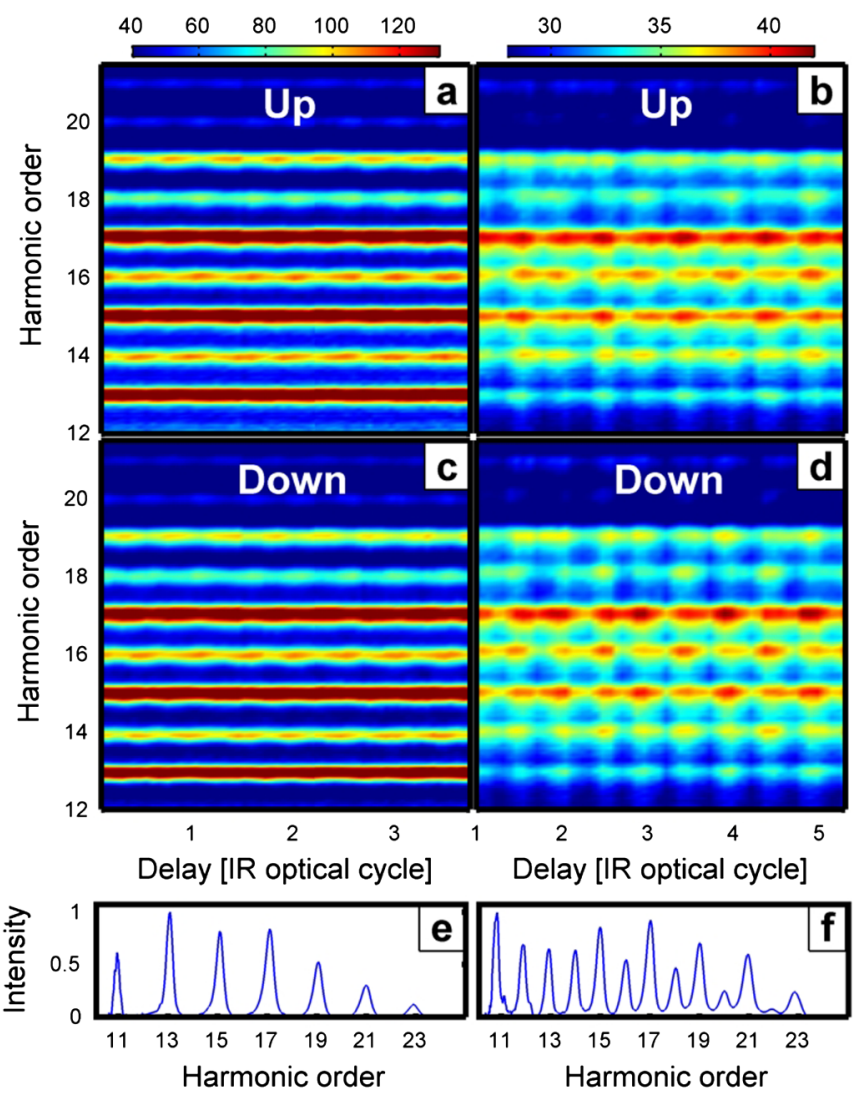

FIG. 2 (color online). Photoelectron energy spectra as a function of the time delay between the IR pulse and the APT formed by odd harmonics only (a),(c), and by both odd and even harmonics (b),(d). (e),(f) Experimental harmonic spectrum. 
time result from the last two of these. Figures 2(b) and 2(d) present the up and down energy spectra as a function of $\tau$ when even and odd harmonics are present. Compared to the RABBITT experiment, new features appear in the photoelectron spectra versus delay. First, oscillations with periods of both 1.35 and $2.7 \mathrm{fs}$ are present in the yields. The longer period is due to the FSI process. Second, the yields of electrons emitted in the up and down directions oscillate out-of-phase with each other, indicating that the emission is now asymmetric along the polarization vector direction.

To better understand these features, we have modeled the angular-resolved photoelectron energy distribution $F_{q}(\theta, \tau)$, within the framework of second-order perturbation theory.
The general full expression obtained by taking into account the final angular momentum of the photoelectrons [21] is not very transparent, and we therefore first choose to discuss the expression which results from the following simplifying assumptions. We consider the initial $m=0$ state only for the $3 p$ electron; we assume that the matrix element from the ground state to the continuum is approximately independent of the final energy and its phase can be factored out; we assume that the continuum-tocontinuum dipole matrix elements are real and equal for single IR photon absorption and emission (none of these assumptions are justified, and we will later relax them). We obtain:

$$
\begin{aligned}
F_{q}(\theta, \tau) \approx & \left|E_{q} e^{i \phi_{q}} f_{e}(\theta)-i \frac{E_{\mathrm{IR}}}{2}\left(E_{q-1} e^{i \phi_{q-1}+i \omega_{0} \tau}+E_{q+1} e^{i \phi_{q+1}-i \omega_{0} \tau}\right) f_{o}(\theta)\right|^{2} \\
\approx & E_{q}^{2} f_{e}(\theta)^{2}+\left(E_{q-1}^{2}+E_{q+1}^{2}\right) \frac{E_{\mathrm{IR}}^{2}}{4} f_{o}(\theta)^{2} \quad \text { DC, } \\
& +E_{q-1} E_{q+1} \cos \left(2 \omega_{0} \tau+\phi_{q-1}-\phi_{q+1}\right) \frac{E_{\mathrm{IR}}^{2}}{2} f_{o}(\theta)^{2} \quad \text { RABB ITT, } \\
& +E_{q}\left[E_{q-1} \sin \left(\omega_{0} \tau+\phi_{q-1}-\phi_{q}\right)-E_{q+1} \sin \left(\omega_{0} \tau+\phi_{q}-\phi_{q+1}\right)\right] E_{\mathrm{IR}} f_{e}(\theta) f_{o}(\theta) \quad \text { FSI, }
\end{aligned}
$$

where $E_{q}$ and $\phi_{q}$ are the magnitude and phase of the $q$ th component of the APT, $E_{\mathrm{IR}}$ and $\omega_{0}$ are the magnitude and angular frequency of the IR field, and $\tau$ the XUV-IR delay. The dipole matrix elements are absorbed into $f_{o}$ and $f_{e}$. Each of these functions is a weighted sum of spherical harmonics with, respectively, odd and even parity and is a real function under our assumptions.

The first term (DC) is the sum of the angular distributions for the one-photon and two-photon processes individually. This term does not depend on $\tau$, and the associated angular distribution can be expanded in even order Legendre polynomials, which are symmetric along the polarization vector direction. The second term is an interference term coming from the cross product of the two-photon transition amplitudes, as observed in the RABBITT experiment. This interference term varies sinusoidally with $\tau$ at twice the IR frequency, and is also symmetric along the polarization vector direction. It too can be expanded in even-order Legendre polynomials. Finally, the last term comes from the cross product of the one- and two-photon transition amplitudes, the FSI process. These interference terms vary sinusoidally with $\tau$ at the IR frequency. Moreover, as expected by simple parity arguments, the angular distribution can be expanded in odd-order Legendre polynomials, which are antisymmetric along the polarization vector direction. To compare our experiment to the prediction of Eq. (1), we have expanded the full angular-resolved photoelectron distribution measured as a sum of odd- and even- Legendre polynomials:

$$
F_{q}(\theta, \tau)=\sum_{J=0}^{2 L \max } \beta_{J}(q, \tau) P_{J}[\cos (\theta)] .
$$

In Fig. 3, we plot the expansion coefficients $\beta_{J}$ associated with the first four Legendre polynomials $P_{J}$ as a function of $\tau$. As expected, the coefficients $\beta_{0}$ and $\beta_{2}$ associated with even-order polynomials oscillate at twice the IR frequency, while coefficients $\beta_{1}$ and $\beta_{3}$ associated with odd-order polynomials oscillate at the IR frequency.

Perhaps the most striking characteristic of these maps is observed in Figs. 3(b) and 3(d): the pattern resembles a checkerboard. This pattern indicates that, at a given time delay $\tau$, the asymmetric emission of photoelectrons associated with odd harmonics points in a direction along the polarization vector opposite to that associated with the even harmonics. Our measurements have shown that this pattern is very robust, insensitive to substantial changes in the relative intensities of the harmonics in the comb. We therefore believe that the origin of this effect lies in the intrinsic relative phases of the consecutive harmonic orders. Under this interpretation, our measurements allow us to determine, from Eq. (1), the phase difference between consecutive odd and even harmonic orders in the comb. Figures 4(a) and 4(b) show the evaluation of the FSI term of Eq. (1) for phase shifts of zero and $\pi / 2$ between even and odd harmonics. We have used harmonic intensities and linewidths consistent with the experimental spectrum, keeping each harmonic phase constant across the harmonic itself. This should be compared to the experimental results of Figs. 3(b) and 3(d). For a phase shift of $\pi / 2$, the agreement with the coefficient $\beta_{1}$ of Fig. 3(b), for which the statistical errors are small, is remarkable. On the contrary, if this shift is set equal to zero [Fig. 4(a)], the checkerboard pattern vanishes.

It would be tempting to conclude from this that the evenodd phase difference is near $\pi / 2$, but closer examination of 


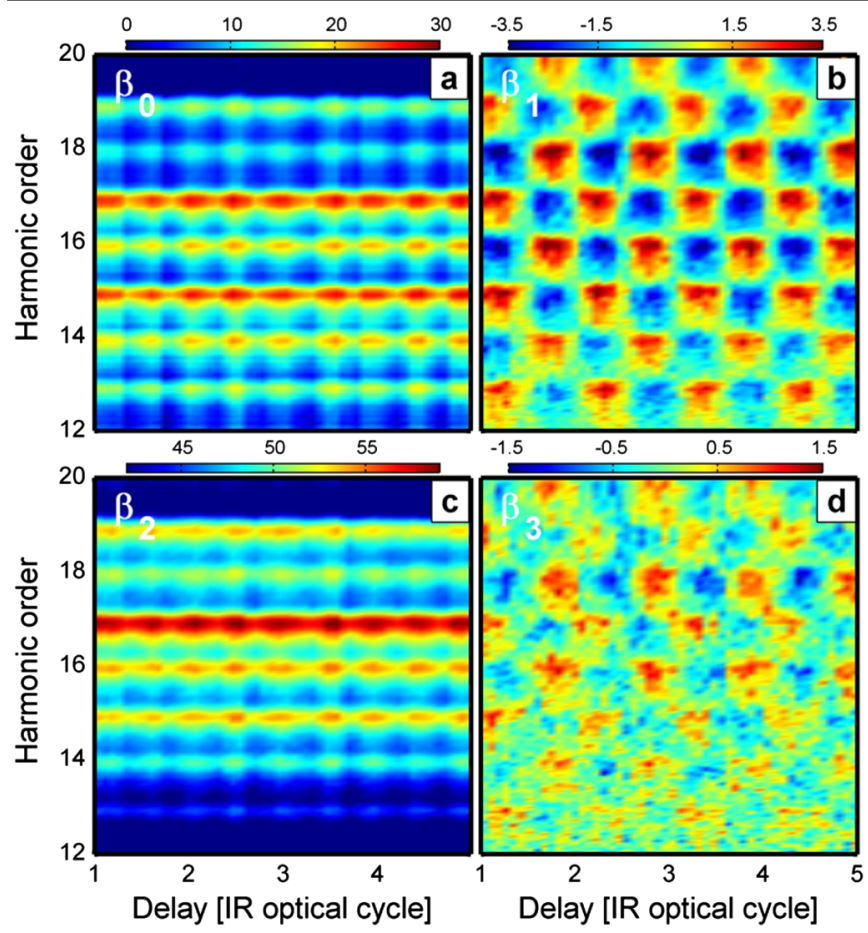

FIG. 3 (color online). Experimental expansion coefficients $\beta_{J}$ associated with the first four Legendre polynomials $P_{J}$.

the FSI term shows that this claim is not justified on the basis of this simplified equation. In fact, any appreciably nonzero phase will yield a checkerboard pattern from this equation. In order to explore this further, we have performed a full calculation, without the simplifying assumptions underlying Eq. (1), of the expected values of $\beta$ coefficients. The bound-free and continuum-continuum matrix elements were calculated using the procedure described in Refs. [22-26]. The calculations were performed for the three possible initial $m$ values for the $3 p$ state and added incoherently. The resulting maps for $\beta_{1}$ are shown in Figs. 4(c) and 4(d) for even-odd phase shifts of $0.35 \pi$ and $0.5 \pi$, respectively. Comparison of Fig. 4(d) with the experimental result of Fig. 3(b) strongly suggests that the actual even-odd phase shift is near $\pi / 2$. On the contrary, Fig. 4(c) represents too large a departure from $\pi / 2$ to be a good representation of the data. The result for $0.65 \pi$ (not shown) is nearly the same as that for $0.35 \pi$, showing that the checkerboard pattern does require a phase near $\pi / 2$.

To our knowledge, this is the first observation of a $\pi / 2$ even-odd phase shift for an attosecond pulse train. We do not yet know of a physical explanation for this phase shift, but the existence of a phase difference between even and odd harmonics in an APT has been discussed theoretically. Zuo et al. predicted, for harmonics near the cutoff, very large phase shifts, nearly $\pi$ [27,28]. Also, a recent calculation of the harmonic generation by a two-color field finds an even-odd phase shift of $\pi / 2$, in exact agreement with the present results [29]. Finally, we emphasize that we have not addressed at all how our even-odd APT is formed, a

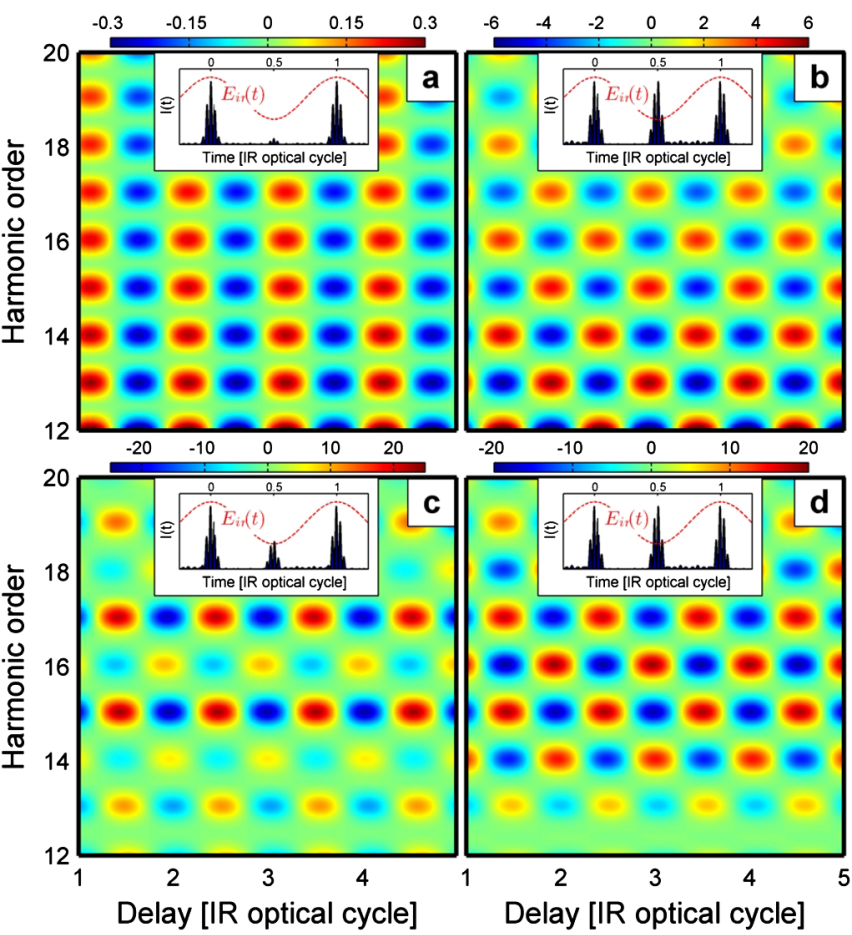

FIG. 4 (color online). Theoretical coefficient $\beta_{1}$ for different even-odd phase shifts. Top row: FSI term for (a) 0 and (b) $0.5 \pi$. Bottom row: Full calculation for (c) $0.35 \pi$ and (d) $0.5 \pi$. Insets: temporal profile of the APTs used in the calculations.

question which has drawn considerable attention in recent years $[15,29-34]$. Our result may be specific to the procedure we have used to generate the APT, with the fundamental and the weak second harmonic fields mainly orthogonally polarized. Further investigation of this question would be a separate, and very worthy, endeavor.

In conclusion, by performing a fully angular-resolved measurement we have explored a process leading to an asymmetric electron emission along the laser polarization direction. The asymmetry can be controlled by varying the time delay between an APT formed by odd and even highorder harmonics and an IR pulse. We have shown that such asymmetric emission is related to the phase of the harmonics in the comb, allowing the measurement of the phase difference between consecutive odd and even harmonics. Our measurements suggest an even-odd phase shift near $\pi / 2$. It is interesting to notice that the resulting APT has a complex structure not resembling a single AP once per IR period.

We thank C. D. Lin for bringing this study to our attention and for illuminating discussions. We thank $\mathrm{M}$. Dahlström and W.-C. Chu for numerous helpful discussions. We thank A. T. Le for providing us with the model phase shifts and radial matrix elements for Ar. The authors wish to thank Professor Z. Chang and his group members for assistance with the laser. This work was supported by three funding agencies. Specifically, (i) the U.S. Army Research Office under Grant No. W911NF-071-0475 
provided the laser, the interferometer, and support for G. L., (ii) the Chemical Sciences, Geosciences, and Biosciences Division, Office of Basic Energy Sciences, Office of Science, U.S. Department of Energy supported the JRML and H.L., I.B. and C.L.C., and (iii) the National Science Foundation under Grant No. CHE0822646 provided the imaging setup and supported W. C. and Z.W.

*glaurent@phys.ksu.edu

[1] G. Sansone, F. Kelkensberg, J. F. Pérez-Torres, F. Morales, M. F. Kling, W. Siu, O. Ghafur, P. Johnsson, M. Swoboda, E. Benedetti, F. Ferrari, F. Lépine, J. L. Sanz-Vicario, S. Zherebtsov, I. Znakovskaya, A. L'Huillier, M. Y. Ivanov, M. Nisoli, F. Martín, and M. J. J. Vrakking, Nature (London) 465, 763 (2010).

[2] K. P. Singh, F. He, P. Ranitovic, W. Cao, S. De, D. Ray, S. Chen, U. Thumm, A. Becker, M. M. Murnane, H.C. Kapteyn, I. V. Litvinyuk, and C. L. Cocke, Phys. Rev. Lett. 104, 023001 (2010).

[3] F. Kelkensberg, W. Siu, J. F. Pérez-Torres, F. Morales, G. Gademann, A. Rouzée, P. Johnsson, M. Lucchini, F. Calegari, J. L. Sanz-Vicario, F. Martín, and M. J. J. Vrakking, Phys. Rev. Lett. 107, 043002 (2011).

[4] P. M. Paul, E. S. Toma, P. Breger, G. Mullot, F. Augé, P. Balcou, H. G. Muller, and P. Agostini, Science 292, 1689 (2001).

[5] J. Itatani, F. Quéré, G. L. Yudin, M. Y. Ivanov, F. Krausz, and P. B. Corkum, Phys. Rev. Lett. 88, 173903 (2002).

[6] Y. Mairesse, A. de Bohan, L. J. Frasinski, H. Merdji, L. C. Dinu, P. Monchicourt, P. Breger, M. Kovačev, R. Taïeb, B.C.H. G. Muller, P. Agostini, and P. Salières, Science 302, 1540 (2003).

[7] R. Kienberger, E. Goulielmakis, M. Uiberacker, A. Baltuska, V. Yakovlev, F. Bammer, A. Scrinzi, T. Westerwalbesloh, U. Kleineberg, U. Heinzmann, M. Drescher, and F. Krausz, Nature (London) 427, 817 (2004).

[8] Y. Mairesse and F. Quéré, Phys. Rev. A 71, 011401(R) (2005).

[9] F. Quéré, Y. Mairesse, and J. Itatani, J. Mod. Opt. 52, 339 (2005).

[10] E. Cormier, I. A. Walmsley, E. M. Kosik, A. S. Wyatt, L. Corner, and L. F. DiMauro, Phys. Rev. Lett. 94, 033905 (2005).

[11] M. Chini, S. Gilbertson, S. D. Khan, and Z. Chang, Opt. Express 18, 13006 (2010).

[12] J. Mauritsson, P. Johnsson, E. Mansten, M. Swoboda, T. Ruchon, A. L'Huillier, and K. J. Schafer, Phys. Rev. Lett. 100, 073003 (2008).
[13] N. N. Choi, T. F. Jiang, T. Morishita, M.-H. Lee, and C. D. Lin, Phys. Rev. A 82, 013409 (2010).

[14] A. T. J. B. Eppink and D. H. Parker, Rev. Sci. Instrum. 68, 3477 (1997).

[15] J. Mauritsson, P. Johnsson, E. Gustafsson, A. L'Huillier, K. J. Schafer, and M. B. Gaarde, Phys. Rev. Lett. 97, 013001 (2006).

[16] I. J. Kim, C. M. Kim, H. T. Kim, G. H. Lee, Y. S. Lee, J. Y. Park, D. J. Cho, and C.H. Nam, Phys. Rev. Lett. 94, 243901 (2005).

[17] L. Brugnera, D. J. Hoffmann, T. Siegel, F. Frank, A. Zaïr, J. W. G. Tisch, and J.P. Marangos, Phys. Rev. Lett. 107, 153902 (2011).

[18] V. Véniard, R. Taïeb, and A. Maquet, Phys. Rev. Lett. 74, 4161 (1995).

[19] V. Véniard, R. Taïeb, and A. Maquet, Phys. Rev. A 54, 721 (1996).

[20] H. Muller, Appl. Phys. B 74, s17 (2002).

[21] E. S. Toma and H. G. Muller, J. Phys. B 35, 3435 (2002).

[22] A.-T. Le, R. R. Lucchese, S. Tonzani, T. Morishita, and C. D. Lin, Phys. Rev. A 80, 013401 (2009).

[23] J. M. Dahlström, Ph.D. thesis, Lund University, 2011 (unpublished).

[24] J. M. Dahlström, T. Fordell, E. Mansten, T. Ruchon, M. Swoboda, K. Klünder, M. Gisselbrecht, A. L'Huillier, and J. Mauritsson, Phys. Rev. A 80, 033836 (2009).

[25] K. Klünder, J. M. Dahlström, M. Gisselbrecht, T. Fordell, M. Swoboda, D. Guénot, P. Johnsson, J. Caillat, J. Mauritsson, A. Maquet, R. Taïeb, and A. L'Huillier, Phys. Rev. Lett. 106, 143002 (2011).

[26] J. M. Dahlström, D. Guenot, K. Klünder, M. Gisselbrecht, J. Mauritsson, A. L'Huillier, A. Maquet, and R. Taïeb, Chem. Phys. (to be published).

[27] T. Zuo, A. D. Bandrauk, M. Ivanov, and P. B. Corkum, Phys. Rev. A 51, 3991 (1995).

[28] M. Ivanov, P. B. Corkum, T. Zuo, and A. D. Bandrauk, Phys. Rev. Lett. 74, 2933 (1995).

[29] J. M. Dahlström, A. L'Huillier, and J. Mauritsson, J. Phys. B 44, 095602 (2011).

[30] N. Dudovich, O. Smirnova, J. Levesque, Y. Mairesse, M. Y. Ivanov, D. M. Villeneuve, and P. B. Corkum, Nature Phys. 2, 781 (2006).

[31] E. Mansten, J. M. Dahlström, P. Johnsson, M. Swoboda, A. L'Huillier, and J. Mauritsson, New J. Phys. 10, 083041 (2008).

[32] J. Mauritsson, J.M. Dahlström, E. Mansten, and T. Fordell, J. Phys. B 42, 134003 (2009).

[33] X. He, J. M. Dahlström, R. Rakowski, C. M. Heyl, A. Persson, J. Mauritsson, and A. L'Huillier, Phys. Rev. A 82, 033410 (2010).

[34] G. Doumy, J. Wheeler, C. Roedig, R. Chirla, P. Agostini, and L. F. DiMauro, Phys. Rev. Lett. 102, 093002 (2009). 